\title{
Reproducibility of optical coherence tomography in vein grafts used for coronary revascularization
}

\author{
Øystein Pettersen ${ }^{1,2}$, Elzbieta Pociask ${ }^{3,4}$, Krzysztof P. Malinowski $^{3,5}$, \\ Magdalena Slezak ${ }^{3}$, Knut Hegbom ${ }^{1}$, Rune Wiseth ${ }^{1,2}$, Dag Ole Nordhaug ${ }^{1,2}$ \\ ${ }^{1}$ Department of Cardiothoracic Surgery Trondheim, St. Olav's University Hospital, Norway \\ ${ }^{2}$ Department of Circulation and Medical Imaging, Faculty of Medicine, \\ Norwegian University of Science and Technology, Trondheim, Norway \\ ${ }^{3}$ Krakow Cardiovascular Research Institute, Krakow, Poland \\ ${ }^{4}$ Department of Biocybernetics and Biomedical Engineering, \\ AGH University of Science and Technology, Krakow, Poland \\ ${ }^{5}$ Institute of Public Health, Faculty of Health Science, \\ Jagiellonian University Medical College, Krakow, Poland
}

\begin{abstract}
Background: Optical coherence tomography (OCT) is a high-resolution imaging modality able to provide near-histological images of vessel walls making it possible to distinguish intima and media layers of the vessel wall separately. The use of this imaging technique is increasing while data on the variability and reliability is lacking. The aim of this study was to investigate the reproducibility of frequency-domain OCT in vein grafts used for coronary revascularization.

Methods: Five pullbacks were analyzed by the same analyst with a 1-month delay (intraobserver) and by two different analysts (interobserver). Five pairs of pullbacks from the same catheters and vein graft were also analyzed (inter pullback).

Results: Optical coherence tomography showed low variability in intra- and interobserver analysis with relative differences of mean media and intima thicknesses and areas of less than 5\% for most parameters. Relative differences of the same parameters in the inter pullback analysis were in the 5-15\% range. Intra- and interobserver reliability was excellent (intraclass correlation coefficient [ICC] >0.90) for intima thickness and intima, media and intima-media area measurements. Inter pullback reliability was good (ICC: 0.75-0.90) for intima and intima-media area measurements, and moderate to good for mean intima thickness measurements (ICC: 0.79; 0.7338-0.8284).

Conclusions: Optical coherence tomography provides good reproducibility for the measurements of parameters relevant for the development of atherosclerosis in vein grafts.

Clinical trial registration: ID NCT01834846. (Cardiol J 2020; 27, 5: 518-523)

Key words: coronary artery disease, saphenous vein graft, intimal hyperplasia
\end{abstract}

\section{Introduction}

Frequency-domain optical coherence tomography (FD-OCT) is a high-resolution intravascular imaging modality that generates near-histological quality in-vivo images of the coronary vessel wall [1]. OCT is being adopted worldwide as an important part of clinical decision-making as well as a promising research tool [2]. Historically, intravascular ultrasound (IVUS) has been the gold

Address for correspondence: Dr. Øystein Pettersen, Department of Cardiothoracic Surgery Trondheim, St. Olav's University Hospital, Norway, tel: + 47 72576791, fax: + 47 72826714, e-mail: oystepet@gmail.com Received: 3.07.2018 Accepted: 2.09.2018 
standard for evaluating the development of intimal hyperplasia in coronary arteries and vein grafts [3-5]. However, IVUS is not able to distinguish between the intima and the media layers of the vessel wall [6]. OCT provides superior resolution by using near-infrared light instead of ultrasound for image acquisition. This provides a more accurate estimate of morphological properties such as lumen diameter [7] and enables researchers to differentiate between the different layers of the vessel wall.

Previous studies have shown excellent intraobserver, interobserver and inter pullback reproducibility for quantitative OCT measurements of lumen diameters and intimal hyperplasia thickness as well as morphometric stent parameters in native coronary arteries [8-10]. Clinical trials using intimal hyperplasia measured with OCT as a marker of development of atherosclerosis in vein grafts are published [11-14]. However, studies investigating the reproducibility of similar parameters in vein grafts used for coronary artery bypass grafting (CABG) are lacking. Different histological morphology and increased diameters in saphenous vein grafts (SVG) compared to native coronary arteries could influence reproducibility of OCT.

\section{Methods}

\section{Study population}

This paper reports reproducibility data from OCT images obtained from patients undergoing CABG using SVG as a conduit for revascularization. The patients were included in a single center randomized trial on SVG harvesting [15]. The patients were examined with OCT 6 months following surgery. The study is registered in Clinicaltrial.org (ID NCT01834846). The study complied with the Consolidated Standards of Reporting Trials (CONSORT) criteria.

\section{Image acquisition}

Optical coherence tomography pullbacks were obtained using commercially available, frequencydomain system (ILUMIEN ${ }^{\mathrm{TM}}$ PCI Optimization System, OCT Intravascular Imaging System; St. Jude Medical, St. Paul, MN, USA). A 2.7 F OCT imaging catheter (Dragonfly; LightLab Imaging, Inc.) was advanced into the vein graft after administration of nitroglycerin $(200 \mu \mathrm{g})$ into the graft. An integrated automated pullback device was used with a speed of $20 \mathrm{~mm} / \mathrm{s}$. The maximal pullback length allowed by the system was $55 \mathrm{~mm}$. The blood was cleared by injection of isoosmolar contrast (Iohexol 350 $\mathrm{mgl} / \mathrm{L}$ Omnipaque, GE Healthcare, Dublin, Ireland) at $37^{\circ} \mathrm{C}$ with an injection pump (ACIST CVi System [ACIST Medical Systems Inc., Eden Prairie, $\mathrm{MN}$, USA]) through the guiding catheter during image acquisition. The size of the vein graft was considered from the angiography and subsequently contrast flow rate and contrast volume given by the Acist system. If the first pullback did not give acceptable pictures, flow rate and volume was adjusted. These parameters were identical between pairs of pullbacks undergoing intra-catheter reproducibility analysis.

All images were digitally stored in the FD-OCT system console and on DVD for later off-line analysis.

\section{Imaging analysis}

Optical coherence tomography analyses were performed at an independent core laboratory (KCRI, Krakow, Poland). OCT pullbacks were analysed using OCT - Ilumien Optis, Offline Review Workstation (St. Jude Medical, USA).

A single, individual analysis comprised of qualitative and quantitative assessment for each graft of interest. OCT analysis was performed according to current consensus standard [16, 17], which focused on measuring thicknesses, areas for intima and media separately. Lumen area was automatically detected and contoured by the software and was manually corrected by the analyst, if necessary. The intima and media contours were delineated for every $1 \mathrm{~mm}$ frame in the region of interest. Frames without clear delineated intima-media border at the entire circumference were excluded from analysis. Intima thickness was defined as the thickness of the high backscattering or signal rich area inside the internal elastic lamina (IEL) in each frame of the pullback. Media thickness was calculated as the mean thickness of the low backscattering area between the IEL and external elastic lamina (EEL) (Fig. 1). Figures 2 and 3 show an example of the maximum difference in media thickness measurements between two separate analyses performed by the same analyst (Fig. 2) and two independent analysts (Fig. 3). For the inter pullback analysis, matching of the frames between the pullbacks were initiated by identifying one corresponding frame visible on both recordings. After finding the corresponding frame, analysis was performed every $1 \mathrm{~mm}$ from this frame, assuring that it covered exactly the same region of interest (the same vessel fragment) as with the previous analysis. 


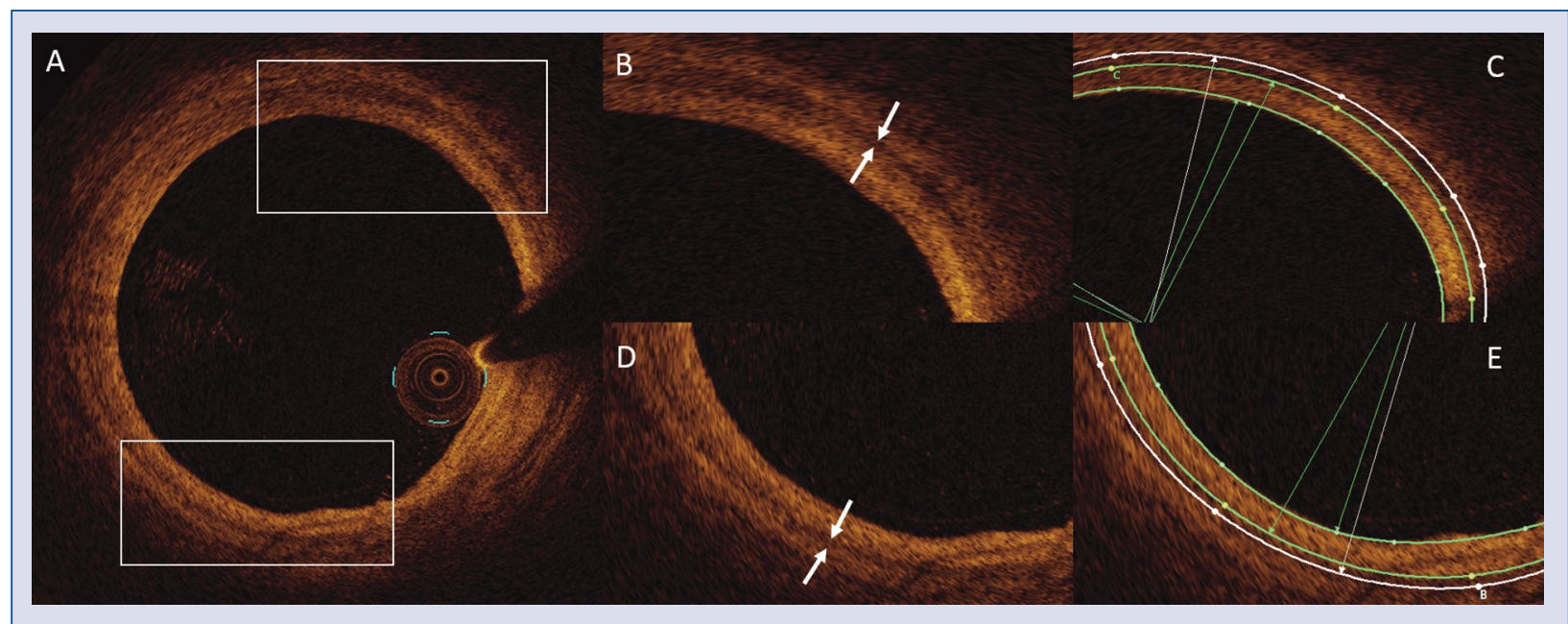

Figure 1. A. Vessel cross-sectional view; B, D. Magnified parts with media (white arrows); C, E. Corresponding frames with lumen (inner green contour), internal elastic lamina (middle green contour) and external elastic lamina (outer white contour) contours. The area between the lumen and the internal elastic lamina is the intima area, whereas the area between the internal and external lamina is the media area.


Figure 2. A. Bland-Altman plots of intraobserver measurements of intima area; B. Bland-Altman plots of interobserver measurements of intima area; C. Bland-Altman plots of inter pullback measurements of intima area.
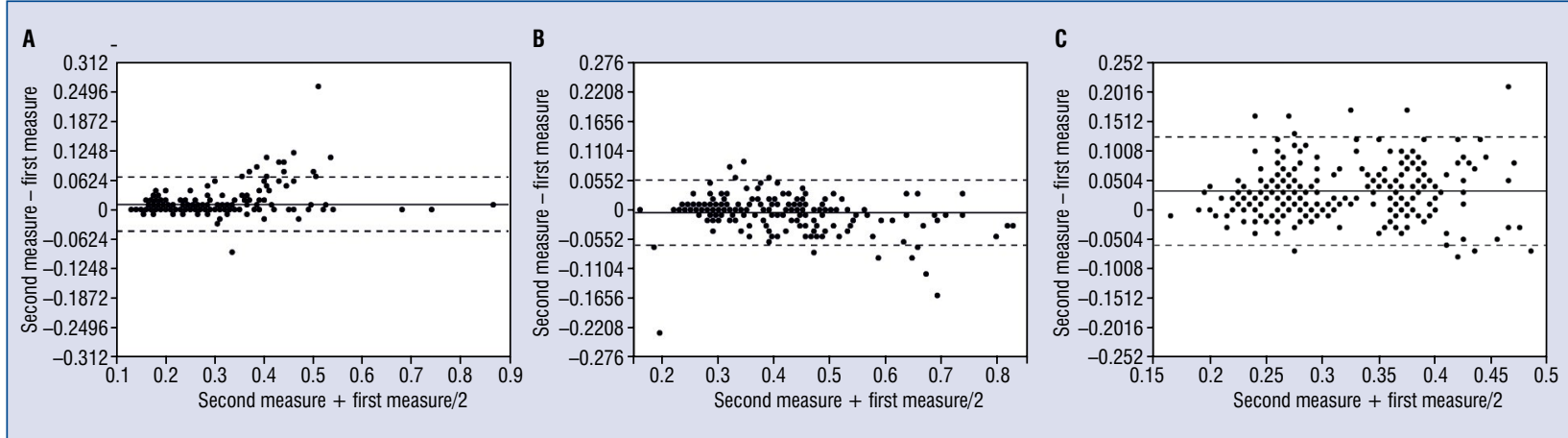

Figure 3. A. Bland-Altman plots of intraobserver measurements of average intima thickness; B. Bland-Altman plots of interobserver measurements of average intima thickness; C. Bland-Altman plots of inter pullback measurements of average intima thickness. 


\section{Statistical analysis}

Five pullbacks with a total of 243 frames were analyzed two times with a 1 month delay on the same software under the same conditions by the same analyst (intra-analysis). Five pullbacks with a total of 258 frames were analyzed correspondingly by two different analysts (inter-analysis). Different frames were analyzed in the interobserver and the intraobserver analysis. Five pullbacks with a total of 258 corresponding frames were analyzed by the same analyst on two different pullbacks obtained from the same OCT catheter and vein graft (inter pullback analysis).

Results of the first and the second measurements were presented as a mean with $95 \%$ confidence interval (CI) and as median with the first and the third quartiles. Normality of distribution was assessed using the Shapiro-Wilk test. Discrepancies between the first and the second analysis were calculated as absolute and relative differences and were presented as means with $95 \%$ CIs. Intraclass correlations were calculated as the main measure of agreement along with the graphical representation as Bland-Altman plots. The intraclass correlation coefficients (ICCs) were calculated based on twoway random effect models $[18,19]$.

\section{Results}

Results of intraobserver, interobserver and inter pullback variability and reliability analysis are presented in Tables 1, 2 and 3, respectively. Bland-Altman plots of interobserver, intraobserver and inter pullback intima area and intima thickness measurements are demonstrated in Figures 2 and 3. The variability of the measurements in the intraand interobserver analysis was low, the relative differences of mean media and intima thicknesses and areas were of less than $5 \%$ for most parameters. Relative differences of the same parameters in the inter pullback analysis were in the $5-15 \%$ range. There were excellent intra- and inter-observer reliability (ICC: $0.90-1.00$ ) for intima and intima-media area as well as diameter measurements. There was good inter pullback reliability on intima and intima-media area measurements (ICC: 0.75-0.90), whereas the mean intima thickness measurements showed moderate to good reliability. The reliability of media thickness measurements was in general poorer in all groups, this is likely due to the absolute thickness being relatively small compared to the other measurements.

\section{Discussion}

Reproducibility of measurements relating to intimal hyperplasia was satisfactory for all variables in the study. The results revealed that albeit satisfactory, the inter pullback reproducibility was inferior to the intra- and interobserver reproducibility. There may be different reasons for this. Pullbacks in the inter pullback analysis were matched frame by frame, however $1 \mathrm{~mm}$ on one pullback does not necessarily correspond to $1 \mathrm{~mm}$ on the other pullback due to cardiac motion during the recording process. Thus, it is possible that the matching of each frame on the pullback did not correspond $100 \%$ with the the previous frames. The absolute difference in small measurements from one frame to the next were small, however relative difference was large, corresponding to a lower ICC than what may be expected.

The data reported here demonstrates the morphological properties of each frame in the vein grafts. Studies reporting vein wall properties are likely to report mean values for segments of vein grafts, not individual frames. This should provide even better inter pullback reliability than that of individual frames, as the problem of matching frames is largely negated. Vein grafts are in general quite large compared to coronary arteries. FD-OCT relies on adequate flushing of blood to achieve acceptable image acquisition. The present experience is that the contrast flow and volume during the pullback must be sufficiently large to provide adequate vein graft flushing during pullback. To obtain this an injection pump for contrast was necessary and several patients received multiple pullbacks of their vein grafts before satisfactory images were acquired. The combination of large lumen, a thickening intima layer and the limited penetration depth associated with OCT are the main limiting factors when visualizing the vein wall.

Reproducibility of longitudinal measurements was not investigated in this study, due to the lack of landmarks for longitudinal measurements. The vein grafts in this study were investigated at 6 months, and as expected there was no evidence of atherosclerotic disease or lesions other than diffuse intimal hyperplasia. The aim of the study was to assess cross sectional vessel wall characteristics. Longitudinal reproducibility should be investigated in vein grafts at a later time point, focusing on atherosclerotic lesion length or stent parameters in vein grafts after undergoing percutaneous coronary intervention. 




\section{Limitations of the study}

The reliability of results presented in this paper are based on a limited number of pullbacks and larger studies are warranted. Studies comparing OCT and IVUS to histological specimen would be ideal in providing assistance to determine a gold standard for imaging morphological development of vein grafts following CABG.

\section{Conclusions}

Optical coherence tomography provides a reliable intraobserver, interobserver and inter pullback assessment of vein graft intimal hyperplasia and other relevant parameters for assessing vein graft morphology. Concluded herein, OCT is a suitable tool for assessing early markers of vein graft disease.

\section{Conflict of interest: None declared}

\section{References}

1. Tearney GJ, Regar E, Akasaka T, et al. Consensus standards for acquisition, measurement, and reporting of intravascular optical coherence tomography studies: a report from the International Working Group for Intravascular Optical Coherence Tomography Standardization and Validation. J Am Coll Cardiol. 2012; 59(12): 1058-72, doi: 10.1016/j.jacc.2011.09.079.

2. Zivelonghi $\mathrm{C}$, Ghione $\mathrm{M}$, Kilickesmez $\mathrm{K}$, et al. Intracoronary optical coherence tomography: a review of clinical applications. J Cardiovasc Med (Hagerstown). 2014; 15(7): 543-553, doi: 10.2459/JCM.0000000000000032.

3. Une D, Kulik A, Voisine P, et al. Correlates of saphenous vein graft hyperplasia and occlusion 1 year after coronary artery bypass grafting: analysis from the CASCADE randomized trial. Circulation. 2013; 128(11 Suppl 1): S213-S218, doi: 10.1161/CIRCULATIONAHA.112.000328, indexed in Pubmed: 24030409.

4. Kaneda H, Terashima M, Takahashi T, et al. Mechanisms of lumen narrowing of saphenous vein bypass grafts 12 months after implantation: an intravascular ultrasound study. Am Heart J. 2006; 151(3): 726-729, doi: 10.1016/j.ahj.2005.05.011, indexed in Pubmed: 16504641.

5. Wakeyama $\mathrm{T}$, Ogawa $\mathrm{H}$, Iida $\mathrm{H}$, et al. Intima-media thickening of the radial artery after transradial intervention. J Am Coll Cardiol. 2003; 41(7): 1109-1114, doi: 10.1016/s0735-1097(03)00089-5.

6. American College of Cardiology Clinical Expert Consensus Document on Standards for Acquisition, Measurement and Reporting of Intravascular Ultrasound Studies (IVUS). A report of the American College of Cardiology Task Force on Clinical Expert Consensus Documents developed in collaboration with the European Society of Cardiology endorsed by the Society of Cardiac Angiography and Interventions. Eur J Echocardiogr. 2001; 2(4): 299-313, indexed in Pubmed: 11908481.

7. Kubo T, Akasaka T, Shite J, et al. OCT compared with IVUS in a coronary lesion assessment: the OPUS-CLASS study. JACC
Cardiovasc Imaging. 2013; 6(10): 1095-1104, doi: 10.1016/j. jcmg.2013.04.014, indexed in Pubmed: 24011777.

8. Antonsen L, Thayssen P, Junker A, et al. Intra- and interobserver reliability and intra-catheter reproducibility using frequency domain optical coherence tomography for the evaluation of morphometric stent parameters and qualitative assessment of stent strut coverage. Cardiovasc Revasc Med. 2015; 16(8): 469-477, doi: 10.1016/j.carrev.2015.08.010, indexed in Pubmed: 26475731.

9. Gerbaud E, Weisz G, Tanaka A, et al. Multi-laboratory interinstitute reproducibility study of IVOCT and IVUS assessments using published consensus document definitions. Eur Heart J Cardiovasc Imaging. 2016; 17(7): 756-764, doi: 10.1093/ehjci/ /jev229, indexed in Pubmed: 26377904.

10. Fedele S, Biondi-Zoccai G, Kwiatkowski P, et al. Reproducibility of coronary optical coherence tomography for lumen and length measurements in humans (The CLI-VAR [Centro per la Lotta contro l'Infarto-VARiability] study). Am J Cardiol. 2012; 110(8): 1106-1112, doi: 10.1016/j.amjcard.2012.05.047, indexed in Pubmed: 22748353.

11. Burris N, Schwartz K, Tang CM, et al. Catheter-based infrared light scanner as a tool to assess conduit quality in coronary artery bypass surgery. J Thorac Cardiovasc Surg. 2007; 133(2): 419-427, doi: 10.1016/j.jtcvs.2006.09.056, indexed in Pubmed: 17258576.

12. Meirson T, Orion E, Di Mario C, et al. Flow patterns in externally stented saphenous vein grafts and development of intimal hyperplasia. J Thorac Cardiovasc Surg. 2015; 150(4): 871-878, doi: 10.1016/j.jtcvs.2015.04.061, indexed in Pubmed: 26242834.

13. Brown EN, Burris NS, Gu J, et al. Thinking inside the graft: applications of optical coherence tomography in coronary artery bypass grafting. J Biomed Opt. 2007; 12(5): 051704, doi: 10.1117/1.2799521, indexed in Pubmed: 17994877.

14. Pettersen $\emptyset$, Wiseth R, Hegbom K, et al. Pedicled vein grafts in coronary surgery exhibit reduced intimal hyperplasia at 6 months. J Am Coll Cardiol. 2016; 68(4): 427-429, doi: 10.1016/j. jacc.2016.04.058, indexed in Pubmed: 27443441.

15. Pettersen $\emptyset$, Haram PM, Winnerkvist A, et al. Pedicled Vein Grafts in Coronary Surgery: Perioperative Data From a Randomized Trial. Ann Thorac Surg. 2017; 104(4): 1313-1317, doi: 10.1016/j.athoracsur.2017.03.076, indexed in Pubmed: 28648540.

16. Prati F, Guagliumi G, Mintz GS, et al. Expert's OCT Review Document. Expert review document part 2: methodology, terminology and clinical applications of optical coherence tomography for the assessment of interventional procedures. Eur Heart J. 2012; 33(20): 2513-2520, doi: 10.1093/eurheartj/ehs095, indexed in Pubmed: 22653335.

17. Prati F, Regar E, Mintz GS, et al. Expert's OCT Review Document. Expert review document on methodology, terminology, and clinical applications of optical coherence tomography: physical principles, methodology of image acquisition, and clinical application for assessment of coronary arteries and atherosclerosis. Eur Heart J. 2010; 31(4): 401-415, doi: 10.1093/eurheartj/ ehp433, indexed in Pubmed: 19892716.

18. Shrout P, Fleiss J. Intraclass correlations: Uses in assessing rater reliability. Psychological Bulletin. 1979; 86(2): 420-428, doi: 10.1037//0033-2909.86.2.420.

19. McGraw K, Wong SP. Forming inferences about some intraclass correlation coefficients. Psychological Methods. 1996; 1(1): 30-46, doi: 10.1037//1082-989x.1.1.30. 